\title{
Article
}

\section{Patient- and parent-initiated oral steroids for asthma exacerbations}

Ganaie, Muhammed, Munawar, M, Gordon, Morris and Evans, David Available at https://clok.uclan.ac.uk/14854/

Ganaie, Muhammed, Munawar, M, Gordon, Morris orcid iconORCID: 00000002-1216-5158 and Evans, David (2016) Patient- and parent-initiated oral steroids for asthma exacerbations. The Cochrane Library . ISSN 1465-1858

It is advisable to refer to the publisher's version if you intend to cite from the work.

For more information about UCLan's research in this area go to

http://www.uclan.ac.uk/researchgroups/ and search for < name of research Group>.

For information about Research generally at UCLan please go to

http://www.uclan.ac.uk/research/

All outputs in CLoK are protected by Intellectual Property Rights law, including Copyright law. Copyright, IPR and Moral Rights for the works on this site are retained by the individual authors and/or other copyright owners. Terms and conditions for use of this material are defined in the policies page.

\section{CLoK}

Central Lancashire online Knowledge www.clok.uclan.ac.uk

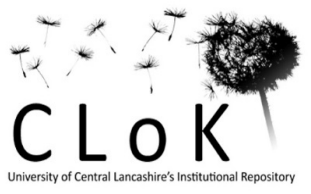




\section{(E) Cochrane Library}

Cochrane Database of Systematic Reviews

\section{Patient- and parent-initiated oral steroids for asthma exacerbations (Protocol)}

Ganaie MB, Munavvar M, Gordon M, Evans DJW

Ganaie MB, Munavvar M, Gordon M, Evans DJW.

Patient- and parent-initiated oral steroids for asthma exacerbations.

Cochrane Database of Systematic Reviews 2016, Issue 5. Art. No.: CD012195.

DOI: 10.1002/14651858.CD012195.

www.cochranelibrary.com 
TABLE OF CONTENTS

HEADER . . . . . . . . . . . . . . . . . . . . . . . . . . . . . . . . . 1

ABSTRACT . . . . . . . . . . . . . . . . . . . . . . . . . . . . . . . . . . . . . . . . . .

BACKGROUND . . . . . . . . . . . . . . . . . . . . . . . . . . . . . . . . . . . .

OBJECTIVES . . . . . . . . . . . . . . . . . . . . . . . . . . . . . . . . . . . . . . . . . . .

METHODS . . . . . . . . . . . . . . . . . . . . . . . . . . . . . . . . . . . . . .

ACKNOWLEDGEMENTS . . . . . . . . . . . . . . . . . . . . . . . . . . . . . . . . . . . . .

REFERENCES . . . . . . . . . . . . . . . . . . . . . . . . . . . . . . . . . . . . . . 6

APPENDICES . . . . . . . . . . . . . . . . . . . . . . . . . . . . . . . . . . . . . . 7

CONTRIBUTIONS OF AUTHORS . . . . . . . . . . . . . . . . . . . . . . . . . . . . . . . . . . . . . . . . . . . . .

DECLARATIONS OF INTEREST . . . . . . . . . . . . . . . . . . . . . . . . . . . . . . . . . . . 9

SOURCES OF SUPPORT . . . . . . . . . . . . . . . . . . . . . . . . . . . . . . . . . . . . . . . . . . . . . . . 


\title{
[Intervention Protocol]
}

\section{Patient- and parent-initiated oral steroids for asthma exacerbations}

\author{
Muhammad B Ganaie $^{1}$, M Munavvar ${ }^{1}$, Morris Gordon ${ }^{2,3}$, David JW Evans ${ }^{4}$ \\ ${ }^{1}$ Respiratory Medicine, Lancashire Teaching Hospitals NHS Foundation Trust, Preston, UK. ${ }^{2}$ School of Medicine and Dentistry, \\ University of Central Lancashire, Preston, UK. ${ }^{3}$ Families Division, Blackpool Victoria Hospital, Blackpool, UK. ${ }^{4}$ Lancaster Patient \\ Safety Research Unit, Royal Lancaster Infirmary, Lancaster, UK
}

Contact address: David JW Evans, Lancaster Patient Safety Research Unit, Royal Lancaster Infirmary, Pointer Court 1, Ashton Road, Lancaster, LA1 4RP, UK. d.evans1@lancaster.ac.uk.

Editorial group: Cochrane Airways Group.

Publication status and date: New, published in Issue 5, 2016.

Citation: Ganaie MB, Munavvar M, Gordon M, Evans DJW. Patient- and parent-initiated oral steroids for asthma exacerbations. Cochrane Database of Systematic Reviews 2016, Issue 5. Art. No.: CD012195. DOI: 10.1002/14651858.CD012195.

Copyright (C) 2016 The Cochrane Collaboration. Published by John Wiley \& Sons, Ltd.

\begin{abstract}
A B S T R A C T
This is the protocol for a review and there is no abstract. The objectives are as follows:

To evaluate the effectiveness and safety of patient- or parent-initiated oral steroids for adults and children with asthma exacerbations.
\end{abstract}

\section{B A C K G R O U N D}

\section{Description of the condition}

Asthma is a chronic condition of the airways affecting an estimated 334 million people worldwide (Global Asthma Report 2014). Direct treatment costs and indirect costs associated with lost productivity are substantial and are among the highest for non-communicable diseases (Global Asthma Report 2014). Asthma triggers may be allergic or non-allergic, resulting in airway inflammation, bronchoconstriction and limitation/obstruction of airflow. During a worsening of asthma symptoms (i.e. an exacerbation), which include tightness of the chest, wheeze and breathlessness, patients will typically exhibit reduced lung function (BTS/SIGN 2014). Impairment of lung function can be reversed with treatment and may return to normal. From a patient perspective, the goals of asthma treatment are to prevent exacerbations, achieve control of daytime and nocturnal symptoms, and permit nor- mal exercise and functional capacity (GINA 2015). Treatment of asthma should be guided by a personalised asthma action plan (GINA 2015), and includes the avoidance of potential triggers, the use of inhaled corticosteroids (ICS) and leukotriene receptor antagonists to reduce airway inflammation, and the use of inhaled long-acting beta 2 -agonists (LABA), short-acting beta 2 -agonists (SABA) and anti-cholinergic bronchodilators (i.e. long-acting muscarinic antagonists (LAMAs)) to relieve airflow limitation (NICE 2007; NICE 2013; BTS/SIGN 2014; GINA 2015). During severe exacerbations, patients may need to attend a medical centre or hospital emergency department for treatment with systemic corticosteroids, which can be administered intravenously or orally (BTS/SIGN 2014; GINA 2015). Some people with asthma are prescribed oral corticosteroids (OCS) for self-administration (i.e. patient-initiated) or to administer to their child with asthma (i.e. parent-initiated), in the event of an exacerbation (Vuillermin 2007). 


\section{Description of the intervention}

Prophylactic treatment with corticosteroids is commonly used in patients with asthma to reduce and control airway inflammation (BTS/SIGN 2014; GINA 2015). ICS are used in preference to systemic corticosteroids because the inhaled dose is delivered directly to the respiratory tract (i.e. drug target), lowering the propensity for systemic side-effects. Adverse effects associated with the longterm use of systemic steroids include: effects on bone density (e.g. osteoporosis and increased risk of femur neck fractures), growth retardation in children, a tendency to hyperglycaemia, and suppression of the response to infection or injury (Rang 2015). Recurrent short courses of prednisolone may also be associated with adverse events, in particular, with a reduction of bone mineral accrual as reported among participants in the Childhood Asthma Management Program (CAMP) trial (CAMP research group 2000; Kelly 2008). However, evidence supports the short-term use of systemic corticosteroids during acute asthma exacerbations (Rowe 2007; Fernandes 2014). Patients remain particularly prone to repeat exacerbations in the period immediately after an asthma exacerbation and the use of systemic steroids can reduce the risk of a relapse and the need for reliever inhalers, without major adverse effects (Rowe 2007). Prescription of a 'rescue-pack' (containing a course of OCS) to a patient or their carer permits self-administered treatment in the event of an exacerbation, as guided by a patient's personalised asthma action plan (BTS/SIGN 2014).

\section{How the intervention might work}

Patient-initiated oral steroids may feature as part of a written asthma action plan (GINA 2015), which should state when and how to initiate treatment with OCS, and when to access medical care if symptoms fail to respond to treatment. Compared with OCS administered by an emergency department physician, patient- or parent-initiated treatment permits early administration of systemic corticosteroids following the onset of an acute exacerbation. The benefits of OCS have been demonstrated within three hours of administration, and delayed dosing of OCS is less effective at resolving acute asthma (Streetman 2002). Indeed, there is some evidence from studies in children that early administration of systemic steroids during an exacerbation can reduce asthma symptoms and the number of days of missed school, compared with physician-initiated steroids (Vuillermin 2010).

\section{Why it is important to do this review}

The use of patient-initiated oral steroids is common practice in chronic obstructive pulmonary disease (COPD) (DoH 2010) and the appropriate use of rescue packs is currently included in a $\mathrm{Na}$ tional Institute for Health and Care Excellence (NICE) quality statement for managing COPD (NICE 2010). In line with their use in COPD, the use of patient- and parent-initiated OCS for asthma appears to be increasingly common in clinical practice (Vuillermin 2007; BTS/SIGN 2014). For example, in an Australian survey of 252 doctors involved in the care of children with asthma, $85 \%$ of doctors reported recommending parent-initiated OCS to parents of children with asthma (Vuillermin 2007). Additionally, BTS/SIGN guidelines on personalised action plan content recommend coverage on starting oral steroids, "which may include provision of an emergency course of steroid tablets" (BTS/SIGN 2014). However, to date there is limited evidence for the use of patient- or parent-initiated OCS for treating asthma exacerbations (NACA 2015). An earlier Cochrane review evaluated the evidence around parent-initiated oral corticosteroid therapy for intermittent wheezing illnesses in children (Vuillermin 2006). We will not consider pre-school wheeze in the present review. It is important to consider the potential benefits of earlier treatment with OCS against potential harms, which include the potential safety issues around delaying access to medical care when OCS are taken at home. Furthermore, the provision of rescue packs to patients with asthma or to their carers will likely increase overall administration of oral corticosteroids; this has implications for the incidence of steroid-associated side-effects, particularly in children. Taken together, this information highlights the importance of synthesising the evidence to establish whether this intervention is safe and effective in people with asthma.

\section{O B JE C T I VES}

To evaluate the effectiveness and safety of patient- or parent-initiated oral steroids for adults and children with asthma exacerbations.

\section{METHODS}

\section{Criteria for considering studies for this review}

\section{Types of studies}

We will include randomised controlled trials (RCTs). We will include studies reported as full-text, those published as abstract only, and unpublished data. We will exclude cross-over trials because the effects of oral corticosteroids can persist for a number of weeks or months (Haahtela 1994) and it is unlikely that studies will employ a sufficient washout period between treatment periods to eliminate cross-over effects. 


\section{Types of participants}

We will include adults (aged $\geq 18$ years) and children of school age or older (i.e. aged $\geq 5$ years) with a diagnosis of asthma. Adults and children will be considered in separate comparisons. Pre-school wheeze will not be considered by this review and as such we will exclude studies of pre-school children. The diagnosis of asthma should be determined by a clinician according to validated national or international guidelines. We will exclude participants with any respiratory co-morbidities (e.g. bronchiectasis, COPD). If a study contains both adults and children, we will contact the study authors to check if disaggregated data are available; if we are unable to source these data we will use the average age ( $\geq 18$ years) of study participants to determine suitability for inclusion. Where the average age of study participants is less than 18 years, we will perform a sensitivity analysis to examine the effect of including or excluding these studies. Finally, if a study includes children of both school and pre-school age, we will exclude the study if the average age is less than five years old.

\section{Types of interventions}

We will include studies comparing any patient- or parent-initiated oral corticosteroid (OCS), with either placebo, normal care, an alternative active treatment plan (e.g. doubling the dose of inhaled steroids) or an identical personalised asthma exacerbation management plan without patient- or parent-initiated OCS. OCS (any dose or duration) can be combined with other measures for the management of an exacerbation (e.g. personalised asthma action plan, increased use of reliever inhaler) provided that the measure is not part of the randomised treatment. We will perform separate comparisons for each type of comparator (e.g. patient-initiated steroids versus placebo; patient-initiated steroids versus normal care, etc); we will also perform separate comparisons for adults and children. We define normal care as any measure that the patient would usually take to manage an exacerbation (e.g. increase reliever inhaler use, seek medical advice, etc). We will also include studies where the comparator group comprises a combination of the above (e.g. personalised asthma action plan plus placebo).

\section{Types of outcome measures}

\section{Primary outcomes}

1. Hospital admissions for asthma

2. Asthma symptoms at follow-up (measured on a validated scale e.g. Asthma Control Questionnaire (ACQ))

3. Serious adverse events

We selected the primary outcomes to represent an important measure of resource use, a patient-reported outcome, and safety.

\section{Secondary outcomes}

1. Unscheduled visit to healthcare provider (e.g. accident and emergency, general practitioner)

2. New exacerbation in follow-up period

3. Asthma control at follow-up

4. Reliever medication use

5. Days off school (children) or study/work (adults) lost

6. Time to full resolution of symptoms

7. Adverse events

Reporting one or more of the outcomes listed here in the study is not an inclusion criterion for the review. If a study uses more than one scale to report the same outcome, or if different scales are used across studies, we will analyse the different scales together using the standardised mean difference (SMD).

\section{Search methods for identification of studies}

\section{Electronic searches}

We will identify trials from the Cochrane Airways Group's Specialised Register (CAGR), which is maintained by the Information Specialist for the Group. The Register contains trial reports identified through systematic searches of bibliographic databases including the Cochrane Central Register of Controlled Trials (CENTRAL), MEDLINE, EMBASE, CINAHL, AMED, and PsycINFO, and handsearching of respiratory journals and meeting abstracts (see Appendix 1 for further details). We will search all records in the CAGR using the search strategy in Appendix 2. We will also conduct a search of ClinicalTrials.gov (www.ClinicalTrials.gov) and the WHO trials portal (www.who.int/ictrp/en/). We will search all databases from their inception to the present, and we will impose no restriction on language of publication.

\section{Searching other resources}

We will check reference lists of all primary studies and review articles for additional references. We will search relevant manufacturers' websites for trial information.

We will search for errata or retractions from included studies published in full-text on PubMed (www.ncbi.nlm.nih.gov/pubmed) and report the date this was done within the review.

\section{Data collection and analysis}

\section{Selection of studies}

Two review authors (MBG, DE) will independently screen titles and abstracts of all the potentially-relevant studies we identify as 
a result of the search and code them as 'retrieve' (eligible or potentially eligible/unclear) or 'do not retrieve'. We will retrieve the full-text study reports/publication and two review authors (MBG, DE) will independently screen the full-text and identify studies for inclusion, and identify and record reasons for exclusion of the ineligible studies. We will resolve any disagreement through discussion or, if required, we will consult a third review author (MM). We will identify and exclude duplicates and collate multiple reports of the same study, so that each study rather than each report is the unit of interest in the review. We will record the selection process in sufficient detail to complete a PRISMA flow diagram and 'Characteristics of excluded studies' table (Moher 2009).

\section{Data extraction and management}

We will use a data collection form for study characteristics and outcome data, which has been piloted on at least one study in the review. Two review authors (MBG, DE) will extract study characteristics from included studies. We will extract the following study characteristics.

1. Methods: study design, total duration of study, details of any 'run in' period, number of study centres and location, study setting, withdrawals, and date of study.

2. Participants: $N$, mean age, age range, gender, severity of condition, diagnostic criteria, baseline lung function, smoking history, inclusion criteria, and exclusion criteria.

3. Interventions: intervention, comparison, concomitant medications, and excluded medications.

4. Outcomes: primary and secondary outcomes specified and collected, and time points reported.

5. Notes: funding for trial, and notable conflicts of interest of trial authors.

Two review authors (MBG, DE) will independently extract outcome data from the included studies. We will note in the 'Characteristics of included studies' table if outcome data were not reported in a usable way. We will resolve disagreements by consensus or by involving a third review author (MM). One review author (DE) will transfer data into the Review Manager (RevMan 2014) file. We will double-check that data are entered correctly by comparing the data presented in the systematic review with the study reports. A second review author (DE) will spot-check study characteristics for accuracy against the trial report.

\section{Assessment of risk of bias in included studies}

Two review authors (MBG, DE) will independently assess risk of bias for each study using the criteria outlined in the Cochrane Handbook for Systematic Reviews of Interventions (Higgins 2011). We will resolve any disagreements by discussion or by involving another review author (MM). We will assess the risk of bias according to the following domains.

1. Random sequence generation.
2. Allocation concealment.

3. Blinding of participants and personnel.

4. Blinding of outcome assessment.

5. Incomplete outcome data.

6. Selective outcome reporting.

7. Other bias.

We will grade each potential source of bias as high, low or unclear risk, and provide a quote from the study report together with a justification for our judgment in the 'Risk of bias' table. We will summarise the 'Risk of bias' judgements across different studies for each of the domains listed. We will consider blinding separately for different key outcomes where necessary (e.g. for unblinded outcome assessment, risk of bias for all-cause mortality may be very different than for a patient-reported pain scale). Where information on risk of bias relates to unpublished data or correspondence with a trialist, we will note this in the 'Risk of bias' table.

When considering treatment effects, we will take into account the risk of bias for the studies that contribute to that outcome.

\section{Assesment of bias in conducting the systematic review}

We will conduct the review according to this published protocol and report any deviations from it in the 'Differences between protocol and review' section of the systematic review.

\section{Measures of treatment effect}

We will analyse dichotomous data as odds ratios (OR) and continuous data as mean difference or standardised mean difference (SMD). We will enter data presented as a scale with a consistent direction of effect.

We will undertake meta-analyses only where this is meaningful, i.e. if the treatments, participants and the underlying clinical question are similar enough for pooling to make sense.

We will narratively describe skewed data reported as medians and interquartile ranges.

Where multiple trial arms are reported in a single trial, we will include only the relevant arms. If two comparisons (e.g. drug A versus placebo and drug B versus placebo) are combined in the same meta-analysis, we will halve the control group to avoid double-counting.

\section{Unit of analysis issues}

For dichotomous outcomes, we will report participants, rather than events, as the unit of analysis. For example, for the primary outcome 'unscheduled visits to the healthcare provider' we would record the number of participants with an unscheduled visit, rather than the number of unscheduled visits per participant. 


\section{Dealing with missing data}

We will contact investigators or study sponsors in order to verify key study characteristics and obtain missing numerical outcome data where possible (e.g. when a study is identified as abstract only). Where this is not possible, and the missing data are thought to introduce serious bias, we will explore the impact of including such studies in the overall assessment of results by conducting a sensitivity analysis.

\section{Assessment of heterogeneity}

We will use the $\mathrm{I}^{2}$ statistic to measure heterogeneity among the studies in each analysis. If we identify substantial heterogeneity we will report it and explore possible causes by prespecified subgroup analysis.

\section{Assessment of reporting biases}

If we are able to pool more than 10 studies, we will create and examine a funnel plot to explore possible small study and publication biases.

\section{Data synthesis}

We will use a random-effects model and perform a sensitivity analysis with a fixed-effect model.

\section{'Summary of findings' table}

We will create a 'Summary of findings' table using the following outcomes: hospital admissions for asthma; asthma symptoms at follow up; serious adverse events; unscheduled visit to healthcare provider; asthma control at follow-up, days off school or study/ work lost, adverse events. We will use the five GRADE considerations (study limitations, consistency of effect, imprecision, indirectness and publication bias) to assess the quality of a body of evidence as it relates to the studies which contribute data to the meta-analyses for the prespecified outcomes (Guyatt 2011). We will use methods and recommendations described in Section 8.5 and Chapter 12 of the Cochrane Handbook for Systematic Reviews of Interventions (Higgins 2011) using GRADEpro software
(GRADEpro GDT). We will justify all decisions to down- or upgrade the quality of studies using footnotes and we will make comments to aid the reader's understanding of the review where necessary.

\section{Subgroup analysis and investigation of heterogeneity}

We plan to carry out the following subgroup analyses.

1. Baseline asthma severity (stratified by background medication)

We will use the following outcomes in subgroup analyses.

1. Hospital admissions for asthma

2. Asthma symptoms at follow-up

3. Serious adverse events

We will use the formal test for subgroup interactions in Review Manager (RevMan 2014).

\section{Sensitivity analysis}

If necessary, we plan to carry out the following sensitivity analyses to explore the effect of including/excluding:

1. Studies that include both adults and children/adolescents, where the average age of participants is $<18$ years

2. Unpublished data (i.e. no peer-reviewed full-text paper available)

3. Studies at high risk of performance or detection bias

4. Studies at high risk of any other bias

5. Studies with missing data

\section{A CKNOW LEDGEMENTS}

The background and methods sections of this protocol are based on a standard template used by Cochrane Airways Group. We thank Elizabeth Stovold for designing the search strategy, and Rebecca Normansell and Emma Welsh for their contribution to the content and methodology of the protocol.

Rebecca Normansell was the contact editor for this protocol and commented critically on the protocol. 


\section{R E F E R E N C E S}

\section{Additional references}

\section{BTS/SIGN 2014}

British Thoracic Society, Scottish Intercollegiate Guidelines Network. British guideline on the management of asthma. Thorax 2014;69(Suppl. 1):1-192.

\section{CAMP research group 2000}

The Childhood Asthma Management Program Research Group. Long-term effects of budesonide or nedocromil in children with asthma. The Childhood Asthma Management Program Research Group. NEJM 2000;343(15):1054-63.

\section{DoH 2010}

Department of Health. Consultation on a strategy for services for chronic obstructive pulmonary disease (COPD) in England. https://www.gov.uk/government/ uploads/system/uploads/attachment_data/file/213840/dh_ 113279.pdf 2010 (accessed 25 November 2015.

Fernandes 2014

Fernandes RM, Oleszczuk M, Woods CR, Rowe BH, Cates CJ, Hartling L. The Cochrane Library and safety of systemic corticosteroids for acute respiratory conditions in children: an overview of reviews. Evidence Based Child Health 2014;9(3):733-47.

\section{GINA 2015}

Global Initiative for Asthma. From the global strategy for asthma management and prevention, Global Initiative for Asthma (GINA) 2015. http://www.ginasthma.org/local/ uploads/files/GINA_Report_2015_Aug11.pdf (accessed 25 May 2015).

Global Asthma Report 2014

Global Asthma Network. The Global Asthma Report 2014. Auckland, New Zealand: Global Asthma Network, 2014. www.globalasthmareport.org/resources/Global_Asthma_ Report_2014.pdf (accessed 20 March 2015).

GRADEpro GDT [Computer program]

GRADE Working Group, McMaster University. GRADEpro GDT. Version accessed 2 February 2016. Hamilton (ON): GRADE Working Group, McMaster University, 2014.

\section{Guyatt 2011}

Guyatt GH, Oxman AD, Kunz R, Vist GE, Falck-Ytter Y, Schunemann HJ. What is "quality of evidence" and why is it important to clinicians. BMJ 2008;336:995-8.

\section{Haahtela 1994}

Haahtela T, Järvinen M, Kava T, Kiviranta K, Koskinen S, Lehtonen $\mathrm{K}$, et al. Effects of reducing or discontinuing inhaled budesonide inpatients with mild asthma. New England Journal of Medicine 1994;331(11):700-5.

Higgins 2011

Higgins JPT, Green S (editors). Cochrane Handbook for Systematic Reviews of Interventions Version 5.1 [updated March 2011]. The Cochrane Collaboration. Available from www.cochrane-handbook.org 2011.
Kelly 2008

Kelly HW, Van Natta ML, Covar RA, Tonascia J, Green RP, Strunk RC. Effect of long-term corticosteroid use on bone mineral density in children: a prospective longitudinal assessment in the childhood Asthma Management Program (CAMP) study. Pediatrics 2008;122:e53-61.

\section{Moher 2009}

Moher D, Liberati A, Tetzlaff J, Altman D. Preferred reporting items for systematic reviews and meta-analyses: the PRISMA statement. PLoS Medicine 2009;6(7): e1000097. [DOI: 10.1371/journal.pmed.1000097]

NACA 2015

National Asthma Council Australia. Guide to systemic corticosteroids. http://www.asthmahandbook.org.au/ resources/medicines-guide/systemic-corticosteroids (accessed 25 November 2015).

NICE 2007

National Institute for Health and Care Excellence. Inhaled corticosteroids for the treatment of chronic asthma in children under the age of 12 years. TA131. www.nice.org.uk/guidance/ta131 2007 (accessed 23 March 2015).

\section{NICE 2010}

National Institute for Health and Care Excellence. Chronic obstructive pulmonary disease in over 16s: diagnosis and management. http://www.nice.org.uk/guidance/cg101/ chapter/1-Guidance\#management-of-exacerbations-ofcopd 2010 (accessed 25 November 2015).

NICE 2013

National Institute for Health and Care Excellence. Quality standard for asthma [QS 25]. www.nice.org.uk/guidance/ qs25 2013 (accessed 23 March 2015).

\section{Rang 2015}

Rang HP, Ritter JM, Flower RJ, Henderson G. Rang and Dale's Pharmacology. 8th Edition. Churchill and Livingstone, 21 January 2015.

RevMan 2014 [Computer program]

The Nordic Cochrane Centre, The Cochrane Collaboration. Review Manager (RevMan). Version 5.3. Copenhagen: The Nordic Cochrane Centre, The Cochrane Collaboration, 2014.

Rowe 2007

Rowe BH, Spooner C, Ducharme F, Bretzlaff J, Bota G. Corticosteroids for preventing relapse following acute exacerbations of asthma. Cochrane Database of Systematic Reviews 2007, Issue 3. [DOI: 10.1002/ 14651858.CD000195.pub2]

\section{Streetman 2002}

Streetman DD, Bhatt-Mehta V, Johnson CE. Management of acute severe asthma in children. Annals of Pharmacotherapy 2002;36:1249-60.

Patient- and parent-initiated oral steroids for asthma exacerbations (Protocol)

Copyright @ 2016 The Cochrane Collaboration. Published by John Wiley \& Sons, Ltd. 
Vuillermin 2006

Vuillermin P, South M, Robertson C. Parent-initiated oral corticosteroid therapy for intermittent wheezing illnesses in children. Cochrane Database of Systematic Reviews 2006, Issue 3. [DOI: 10.1002/14651858.CD005311.pub2]

Vuillermin 2007

Vuillermin PJ, South M, Carlin JB, Biscan MI, Brennan SL, Robertson CF. Parent-initiated oral corticosteroid therapy for acute asthma: a survey of current practice. Journal of Paediatrics and Child Health 2007;43(6):443-5.

Vuillermin 2010

Vuillermin PJ, Robertson CF, Carlin JB, Brennan SL, Biscan MI, South M. Parent initiated prednisolone for acute asthma in children of school age: randomised controlled crossover trial. BMJ 2010;340:c843.

* Indicates the major publication for the study

\section{A P P E N D I C E S}

\section{Appendix I. Sources and search methods for the Cochrane Airways Group's Specialised Register (CAGR)}

Electronic searches: core databases

\begin{tabular}{ll}
\hline Database & Frequency of search \\
\hline CENTRAL (Cochrane Library) & Monthly \\
\hline MEDLINE (Ovid) & Weekly \\
\hline EMBASE (Ovid) & Weekly \\
\hline PsycINFO (Ovid) & Monthly \\
\hline CINAHL (EBSCO) & Monthly \\
\hline AMED (EBSCO) & Monthly \\
\hline
\end{tabular}

Handsearches: core respiratory conference abstracts 


\begin{tabular}{l|l}
\hline Conference & Years searched \\
\hline American Academy of Allergy, Asthma and Immunology (AAAAI) & 2001 onwards \\
\hline American Thoracic Society (ATS) & 2001 onwards \\
\hline Asia Pacific Society of Respirology (APSR) & 2004 onwards \\
\hline British Thoracic Society Winter Meeting (BTS) & 2000 onwards \\
\hline Chest Meeting & 2003 onwards \\
\hline European Respiratory Society (ERS) & $1992,1994,2000$ onwards \\
\hline International Primary Care Respiratory Group Congress (IPCRG) & 2002 onwards \\
\hline Thoracic Society of Australia and New Zealand (TSANZ) & 1999 onwards \\
\hline
\end{tabular}

\section{MEDLINE search strategy used to identify trials for the CAGR}

\section{Asthma search}

1. exp Asthma/

2. asthma\$.mp.

3. (antiasthma $\$$ or anti-asthma $\$$ ).mp.

4. Respiratory Sounds/

5. wheez\$.mp.

6. Bronchial Spasm/

7. bronchospas $\$ . m p$.

8. (bronch $\$$ adj3 spasm\$).mp.

9. bronchoconstrict $\$ . \mathrm{mp}$.

10. exp Bronchoconstriction/

11. (bronch\$ adj3 constrict\$).mp.

12. Bronchial Hyperreactivity/

13. Respiratory Hypersensitivity/

14. ((bronchial\$ or respiratory or airway $\$$ or lung $\$$ ) adj3 (hypersensitiv\$ or hyperreactiv\$ or allerg $\$$ or insufficiency)).mp.

15. ((dust or mite\$) adj3 (allerg\$ or hypersensitiv\$)).mp.

16. or/1-15

\section{Filter to identify RCTs}

1. exp "clinical trial [publication type]"/

2. (randomized or randomised).ab,ti.

3. placebo.ab,ti.

4. dt.fs.

5. randomly.ab,ti.

6. trial.ab,ti.

7. groups.ab,ti. 
8. or/1-7

9. Animals/

10. Humans/

11. 9 not (9 and 10)

12. 8 not 11

The MEDLINE strategy and RCT filter are adapted to identify trials in other electronic databases.

\section{Appendix 2. Search strategy to identify relevant trials from the CAGR}

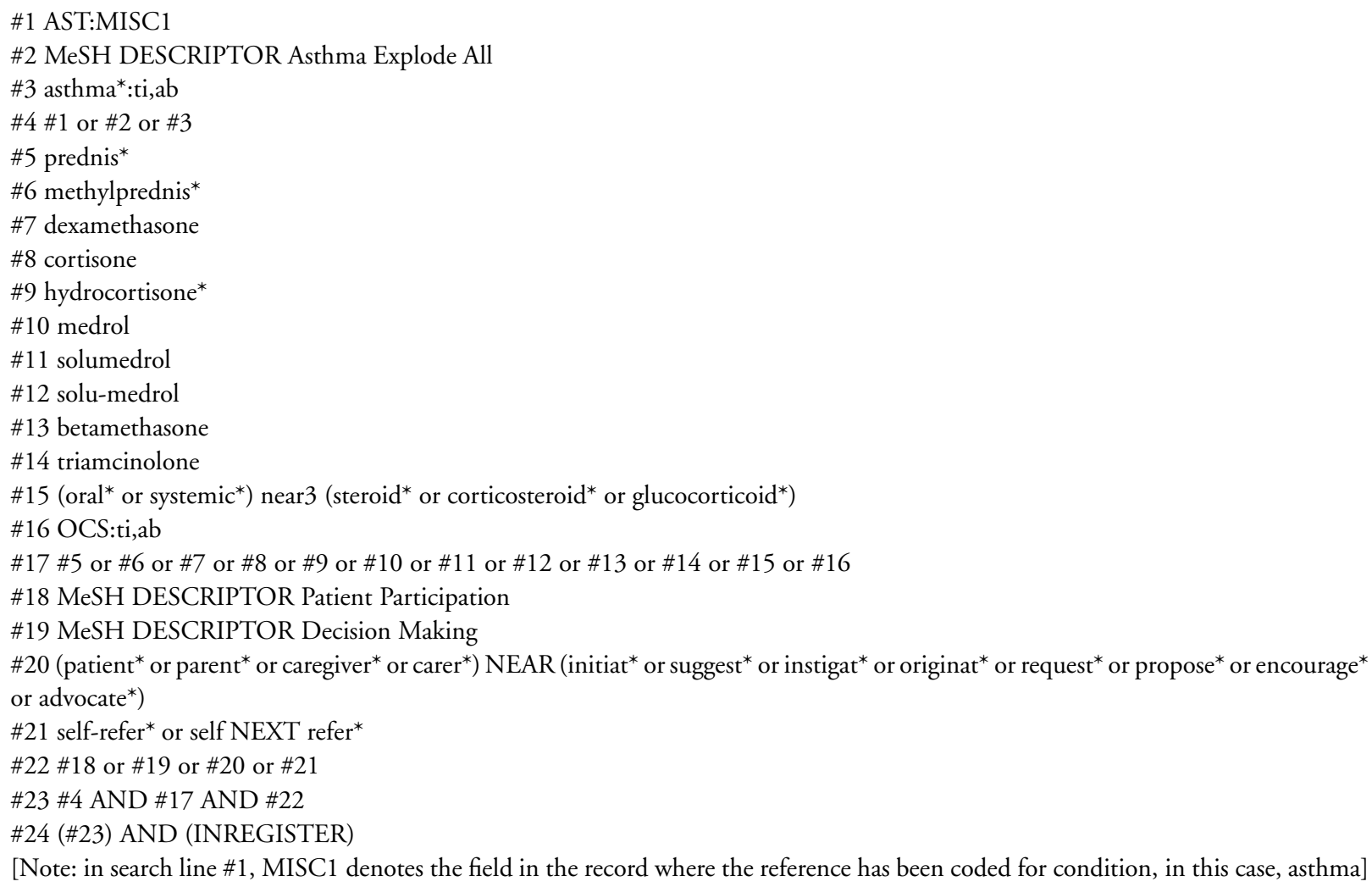

\section{CONTRIBUTIONSOFAUTHORS}

All review authors contributed to the drafting of the protocol, reviewed it critically for intellectual content, provided final approval of the version to be published and are accountable for all aspects of the work.

\section{DECLARATIONSOF INTEREST}

MB Ganaie: none

D Evans: provides freelance writing services to medical communication agencies.

M Munavvar: none

M Gordon: none 


\section{SOURCES OF SUPPORT}

\section{Internal sources}

- National Institute for Health Research, UK.

David Evans: Evidence to guide care in adults and children with asthma, 13/89/14.

\section{External sources}

- National Institute for Health Research (NIHR), UK.

This project was supported by the National Institute for Health Research (NIHR), via Cochrane Infrastructure, Cochrane Programme Grant or Cochrane Incentive funding to the Airways Group. The views and opinions expressed herein are those of the authors and do not necessarily reflect those of the Systematic Reviews Programme, NIHR, NHS or the Department of Health 